\title{
Evolution of Dynamic Localization Regimes in Coupled Minibands
}

\author{
P. H. Rivera ${ }^{1 *}$ and P. A. Schulz ${ }^{2}$ \\ ${ }^{1}$ Departamento de Física, Universidade Federal de São Carlos \\ Caixa Postal 676, 13564-200, São Carlos, SP, Brazil \\ ${ }^{2}$ Instituto de Física Gleb Wathagin, Universidade Estadual de Campinas \\ Caixa Postal 6165, 13083-970, Campinas, SP. Brazil
}

Received February 7, 1999

\begin{abstract}
We consider the behaviour of two coupled symmetric electronic minibands in the presence of an AC intense field. Symmetric coupled minibands are realized as dimerization procedures. The minibands are emulated by a tight-binding finite chain, whereas the interaction with an AC field is described by a time-independent Hamiltonian. We follow the evolution of dressed minibands, non perturbatively, in a wide frequency range. For one dimerization procedure, a transition between both limits can be systematically followed and a dynamic breakdown is unambigously identified. The most striking effect that may occur is a field-induced analog of an insulator-metal transition due to the suppression of a Peierls-like instability.
\end{abstract}

The electronic properties of semiconductor microstructures dressed by intense AC fields have been attracting growing interest in the past few years, leading to predictions of tunable quantum well lasers [1], dynamic localization (DL) in dressed minibands of semiconductor superlattices $[2,3]$, as well as resonant tunneling suppression [4]. These field effects on superlattices have been observed experimentally [5, 6]. One important theoretical result is that DL in isolated minibands can be characterized by miniband collapses obeying the zero order Bessel function (ZOBF) roots, where the argument of $J_{0}$ involves the field frequency and intensity. The present work is concerned with the intense AC field effects on coupled superlattice minibands. Although intensively studied in the past few years $[7,8,9,10]$, there are still open questions related to the mechanisms of DL in multi-miniband systems.

One of these multiminibands system is a set of two coupled symmetric minibands, completely isolated from other minibands. They are obtained by either one of two dimerization processes, as was suggested by Holthaus[7]: alternating two barrier widths or alternating two different quantum wells.

We start our work by simulating these dimerized superlattices with a heuristic tight-binding finite chain model. For two alternating barrier widths, the Hamiltonian is given by:

$$
\begin{gathered}
H_{o}=E_{0} \sum_{\ell m}|\ell m><\ell m|+ \\
\frac{1}{2} \sum_{\ell n}\left\{\left[V_{1} \frac{(-1)^{\ell}+1}{2}+V_{2} \frac{(-1)^{\ell+1}+1}{2}\right] \times\right. \\
|\ell m><\ell+1, m|+\left[V_{1} \frac{(-1)^{\ell+1}+1}{2}+V_{2} \frac{(-1)^{\ell}+1}{2}\right] \times \\
|\ell+1, m><\ell m|\} .
\end{gathered}
$$

And for two alternating quantum wells, we have

$$
\begin{gathered}
H_{0}=\sum_{\ell m}\left[E_{o}+(-1)^{\ell} \Delta\right]|\ell m><\ell m|+, \\
\frac{V}{2} \sum_{\ell m}[|\ell m><\ell+1, m|+|\ell+1, m><\ell m|]
\end{gathered}
$$

where $V_{1}, V_{2}, V$ are the hopping parameters related to the barrier widths; $\ell, m$ are the site and the photon index, respectively. $E_{0}$ is the s-orbital energy related to the quantum well state energy and $\Delta$ the difference of energy with respect to the first neighbor s-orbital energy, leading to $E_{g}=2 \Delta$ when the intensity of the field is zero.

The interaction with an intense $\mathrm{AC}$ field of a given frequency is described by a semiclassic time-dependent

*pablo@lab.df.ufscar.br; pablo@ifi.unicamp.br 
Hamiltonian, which is replaced by a infinite timeindependent Hamiltonian through a Floquet-Fourier unitary tranformation. The treatment of the timedependent Schrödinger equation is based on Floquet states $\mid \ell, m>$ following the procedure developed by Shirley[11]. The so called called Floquet matrix for the case of two alternating quantum wells is given by

$$
\begin{gathered}
\left(\mathcal{E}-m \hbar \omega-\left[E_{o}+(-1)^{\ell} \Delta\right] \delta_{\ell^{\prime} \ell} \delta_{m^{\prime} m}=\right. \\
\frac{V}{2} \delta_{m^{\prime} m}\left(\delta_{\ell^{\prime}, \ell-1}+\delta_{\ell^{\prime}, \ell+1}\right)+ \\
\frac{1}{2} e a F \ell \delta_{\ell^{\prime} \ell}\left(\delta_{m^{\prime}, m-1}+\delta_{m^{\prime}, m+1}\right),
\end{gathered}
$$

where $\mathcal{E}$ is the quasi-energy spectrum of the system, a new motion constant due to the fact that a new quasi Brillouin zone (QBZ) created by the photon field is associated to the system as well as to the photon energies. $F, \omega$ are the intensity and angular frequency of the AC field, respectively; $a$ is the SL period, and $e$ is the electron charge.

The dimension of the matrix is given by $L(2 M+1)$, where $L$ is the number of sites, while $M$ is the maximum of the photon index, given by a convergence criterium: no level crossing at the QBZ edge, being the first one spanned in the range $-\hbar \omega / 2 \leq \mathcal{E} \leq \hbar \omega / 2$ [2].

In what follows we represent the eigenvalues of the Floquet matrix (quasi-energies) versus the intensity of the electric field, in units of potential drop by a period of the superlattice, $e a F$. We consider AC external fields with wavelengths that are many times longer than the length of the superlattice and all are linearly polarized along the superlattice growth direction. The frequency will be constant along the present analysis, $\hbar \omega=0.5$ $\mathrm{meV}$, but the tight-binding parameters will be varied throughout the work.

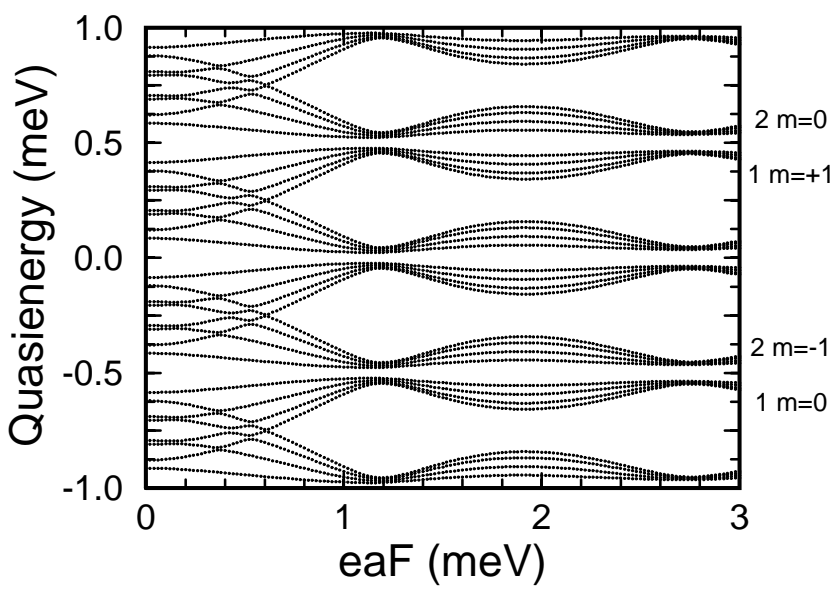

Figure 1. Dressed Minibands corresponding to a linear chain with $L=8, V=0.2 \mathrm{meV}, E_{g}=0.1 \mathrm{meV}, M=40$. We observe a DL similar to an isolated miniband.
We first analyse a dimerized superlattice obtained by alternating two different quantum wells. In Fig.1 the case for high frequency limit, i.e. when $E_{g} \ll \hbar \omega$, is shown. Here the case of $M=8$ sites with a hopping parameter $V=0.2 \mathrm{meV}, E_{g}=0.1 \mathrm{meV}$ and $L=40$ photons is simulated. The number $x m= \pm m$ in Fig. 1 and others represent the position in energy of the dressed miniband $x=1$ (fundamental) or $x=2$ (excited) due to the absorption (+) or emission (-) of $m$ photons.

The DL of replicas of the two minibands obeys to the ZOBF roots distribution $\left(J_{0}(e a F / \hbar \omega)\right)$ and the memory of the DL of replicas of an isolated miniband, $E_{g}=0 \mathrm{meV}$, remains[12]. This type of regime is valid for low and high intensities of $\mathrm{AC}$ field and this behaviour is predicted by an analytical equation obtained through the gauge transformation $\hbar k \longrightarrow \hbar k+e A(t)$ $[2,3]$ also discussed by Zhao[9]:

$$
\mathcal{E}(k, \omega)= \pm 2\left[E_{g}^{2}+V^{2} J_{0}^{2}\left(\frac{e a F}{\hbar \omega}\right) \cos ^{2}\left(\frac{k a}{2}\right)\right]^{1 / 2} .
$$

By varying the tight-binding parameters, the system will evolve from high to low frequencies even when the AC field frequency remains constant. As $E_{g}$ increases, the collapses mentioned above disappear and the miniband replicas evolves to a shape where other different collapses begin to appear.

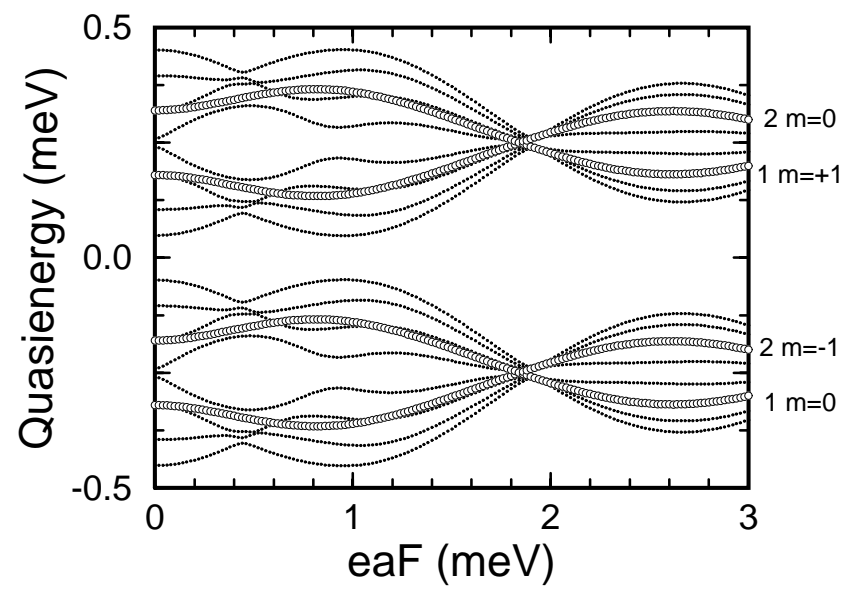

Figure 2. Dressed minibands corresponding to a linear chain with $L=8, V=0.2 \mathrm{meV}, E_{g}=0.5 \mathrm{meV}$ and $M=40$ (dots).Two level system $L=2$ with same parameters (circles).

The case $E_{g}=\hbar \omega=0.5 \mathrm{meV}$ is very interesting to observe, Fig.2. Here, at low intensities there is a collapse very similar to DL of replicas of an isolated miniband where the ZOBF roots are now associated to the argument $e(2 a) F / \hbar \omega$. However, due to dynamical Stark effect at high field intensities the two minibands 
collapses in a way resembling the corresponding two level system (single pair of sites). At these collapses the dimerized superlattice miniband behaves as an isolated set of dimers. At intermediate field intensities the miniband width is greater and a dynamic breakdown occurs, separating the two limits described above. These characteristics are better observed in Fig.3, for $E_{g}=2.0 \mathrm{meV}$. At low intensities, the DL of isolated minibands replicas are well defined, at $e a F \approx 0.6,1.3$ meV, related to zeros of $J_{0}(e(2 a) F / \hbar \omega)$. At high intensities there are collapses of dressed isolated dimers, $e a F \approx 3.8 \mathrm{meV}$; while a dynamic breakdown of the dressed minibands occurs at $e a F \approx E_{g}$. Just beyond the dynamic breakdown line a first induced analog of an insulator-metal transition due to total suppression of a Peierls-like instability may be observed.

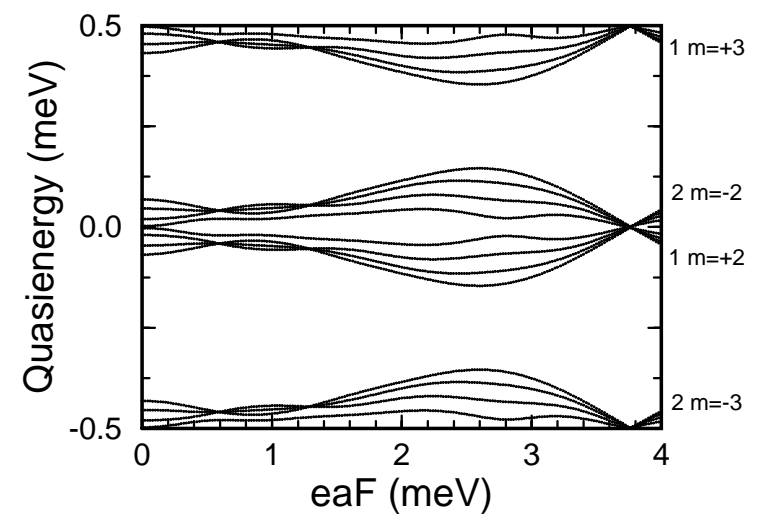

Figure 3. Dressed minibands corresponding to a linear chain with $L=8, V=0.2 \mathrm{meV}, E_{g}=2.0 \mathrm{meV}$ and $M=40$. There are two DL of isolated minibands at $e a F \approx 0.6,1.3$ $\mathrm{meV}$, and a collapse of the minibands at $e a F \approx 3.8 \mathrm{meV}$, and a induced field analog of an insulator-metal transition at $e a F \approx 2.8 \mathrm{meV}$.

The many aspects discussed above can be better visualized in a density of states (DOS) representation, Fig.4. Here we start from the spectra of dressed minibands for a linear chain with $L=20$ and $M=90$, while the other parameters are the same as in Fig.3. The DOS of dressed minibands are spanned in the first QBZ. In Fig.4.(a), the DOS related to coupled miniband replicas when they are collapsing like dressed isolated minibands (low field intensity) is shown. The surface states are in the gap between both miniband replicas, as can be seen in the figure. In Fig.4.(b), the DOS in the the region of dynamic breakdown of the miniband replicas, at $e a F=2.2 \mathrm{meV}$, is represented. The minibands have lost their isolated superlattice miniband identity and begin to predominate their dimer identity. Signatures of an insulator-metal transition, induced dynamically by the AC field, is show in Fig.4.(c) at $e a F=2.8 \mathrm{meV}$. Here a clear single band like DOS can be observed, revealing the suppression of the originally imposed dimer- ization for this particular field intensity. This analog to a Peierls instability is supressed at certain field intensities, whenever $e a F>E_{g}$ as in Fig.4.(c), i.e., the $\mathrm{AC}$ field reduces the gap, dynamically leading to equal effective barrier widths and equal effective quantum wells. Finally, Fig.4.(d), shows the DOS of the collapse of all dimers at $e a F=3.76 \mathrm{meV}$.

Clearly we observe two different regimes of DLs, when $E_{g} \geq \hbar \omega$. At low intensities, we have the DL of isolated minibands with the related collapses remaining for any value $E_{g} \geq \hbar \omega$. At high intensities, there are collapses of dimers only when $E_{g} / \hbar \omega=n, n$ is integer. Out of this condition the dimer collapses evolve to a double collapse structure that remains for high field intensities, Fig.5.

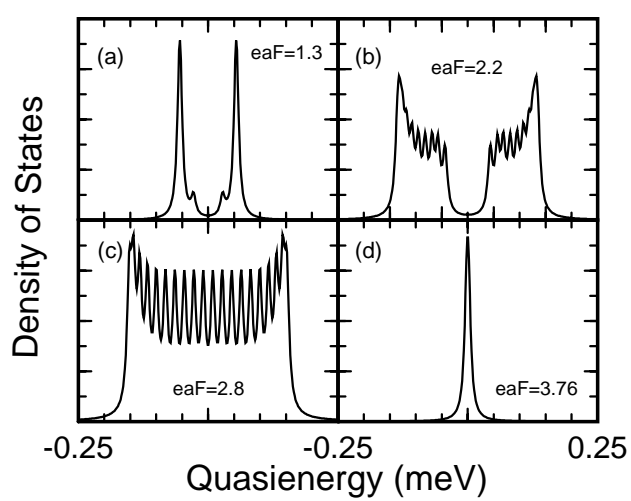

Figure 4. The DOS for a linear chain with $L=20, V=0.2$, $E_{g}=2.0 \mathrm{meV}, \hbar \omega=0.5 \mathrm{meV}$ and $M=90$ is shown. (a) DL of isolated minibands at $e a F=1.3 \mathrm{meV}$; (b) dynamic breakdown of the minibands at $e a F=2.2 \mathrm{meV}$; (c) transition to single-band DOS induced by the field at $e a F=2.8$ $\mathrm{meV}$; and (d) collapse of the dimers at $e a F=3.76 \mathrm{meV}$.

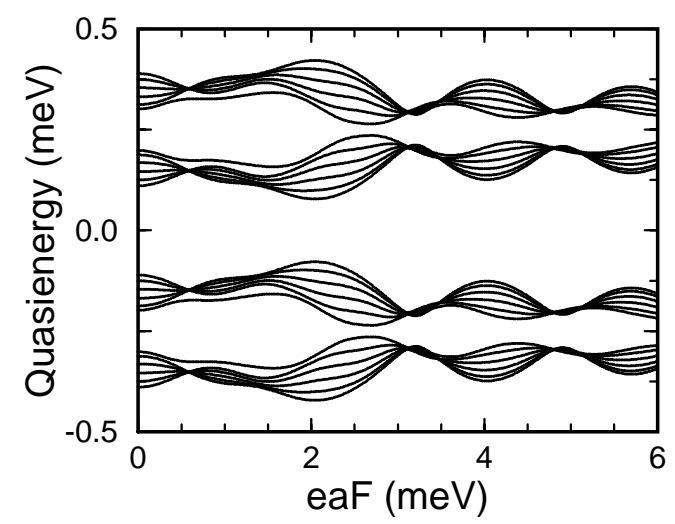

Figure 5. Dressed minibands corresponding to a linear chain with $L=8, V=0.2 \mathrm{meV}, E_{g}=1.6 \mathrm{meV}$ and $M=40$ photons. The system is out of resonance condition $E_{g} / \hbar \omega=n$ and a double structure of dimer collapses for high intensities is observed. 
The dimerization by alternating the barriers leads to a more involved physical picture, which has been only partially addressed. The model for this situation is implemented by simply alternating the hopping parameters, while keeping the atomic site orbital energy fixed throughout the linear chain, Eq.(1). In the high frequency limit an analytical exprression for the dressed spectra is obtained in a similar way as for the previous case. Here:

$$
\mathcal{E}\left(k_{z}\right)= \pm \sqrt{\left(V_{1}^{2}+V_{2}^{2}\right)+2 V_{1} V_{2} \cos \left(k_{z} a\right)}\left|J_{0}\left(\frac{e a F}{\hbar \omega}\right)\right|
$$

where the gap, in function of the field intensity, is defined by $E_{g}=2\left|V_{1}-V_{2}\right| J_{0}(e a F / \hbar \omega)$ and the miniband width by $\Delta \mathcal{E}=2 V_{1}$, considering that $\left|V_{2}>V_{1}\right|$.

This analytical solution [10] is indeed a good approximation for the numerical results, as shown in Fig.6. Comparying with Fig.1, we already see that both dimerization procedures, although leading to qualitatively similar electronic structures in absence of $\mathrm{AC}$ fields, imply in completely different behaviours for the corresponding dressed spectra.

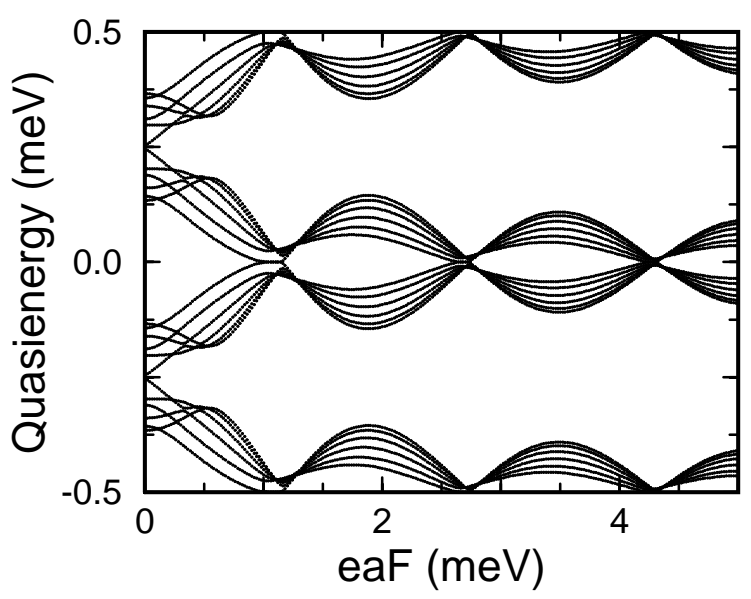

Figure 6. Dressed minibands of a linear chain with $L=12$ and $M=60 ; V_{1}=0.125 \mathrm{meV}$ and $V_{2}=0.25 \mathrm{meV}$, and $\hbar \omega=0.5 \mathrm{meV} . E_{g}=0.25 \mathrm{meV}$ and $\Delta \mathcal{E}=0.25 \mathrm{meV}$ at $e a F=0$.

In the scenario given by this second dimerization procedure, the interplay between intra and inter miniband coupling is of paramount importance. In the low frequency limit, isolated miniband behaviour as a function of field intensity may be clearly identified, whenever the miniband width is less than the field frequency. On the other hand, signatures of this behaviour survive for very high field intensities, as can be seen in Fig. 7 . In contrast to what has been shown for the dimerization by alternating quantum wells, no clear dynamic breakdown intensity region can be traced and no clear resemble of a two level system is found. Actual superlattices should show characteristics involving both procedures discussed in this work. Further work is necessary in order to verify how robust the features analysed here, like the insulator-metal transition, as well as the dynamic breakdown, are in front of deviations of the ideal dimerization conditions.

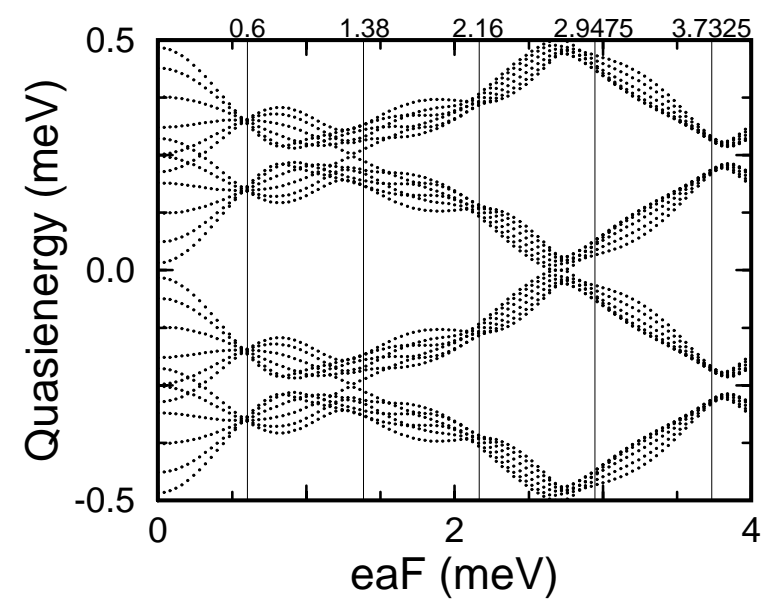

Figure 7. Dressed minibands of linear chain with $L=12$ and $M=60 . V_{1}=0.15, V_{2}=1.15 \mathrm{meV}$, and $\hbar \omega=0.5$ meV. $E_{g}=2.0 \mathrm{meV}$ and $\Delta \mathcal{E}=0.3 \mathrm{meV}$ at $e a F=0$. The vertical lines indicate the $\mathrm{ZOBF}$ roots.

In conclusion, in dimerized SL for alternating quantum wells there are three different dynamic localization. As isolated minibands when $\hbar \omega<E_{g}$ and as non coupled minibands for $\hbar \omega>E_{g}$. The other regime resemble to dimer collapses for $\left(\hbar \omega / E_{g}\right)=n<\left(e a F / E_{g}\right)$ and a field induced metal-insulator like transition due to the break of like Peierls instability is observed. Also a dynamic breakdown occur at $e a F \approx E_{g}$.

Although the observability of these effects deserves further work, the present results are for parameters that resemble typical semiconductor systems. The frequency $\hbar \omega=0.5 \mathrm{meV}$ corresponds to $0.1 \mathrm{THz}$, while miniband dispersion and gap of the order of meV or less are typical for GaAs/AlGaAs superlattices with periods of a few hundred Angstroms, corresponding to field intensities, as shown in the figures, of the order of $10-100$ $\mathrm{kV} / \mathrm{cm}$, which are usual values [7].

The authors acknowledge the financial supports of Fundação de Amparo à Pesquisa do Estado de São Paulo (FAPESP) and Conselho Nacional de Desenvolvimento Científico e Tecnológico (CNPq).

\section{References}

[1] E. Gerck and L. C. M. Miranda, Appl. Phys. Lett. 44 , 837 (1984)

[2] M. Holthaus, Phys. Rev. Lett. 69, 351 (1992) 
[3] M. Holthaus, Z. Phys. B 89, 251 (1992)

[4] M. Wagner, Phys. Rev. B 49, 16544 (1994)

[5] P.S.S. Guimaraes et al, Phys. Rev. Lett. 70, 3792 (1993)

[6] B. J. Keay et al, Phys. Rev. Lett. 75, 4102 (1995)

[7] D. Hone and M. Holthaus, Phys. Rev. B 48, 15123 (1993)

[8] K. Drese and M. Holthaus, J. Phys.: Cond. Matter 8, $1193(1996)$
[9] X-G. Zhao, J. Phys.: Cond. Matter 9, L385 (1997)

[10] S-Q. Bao, X-G. Zhao, X-W. Zhang, W-X. Yan, Phys. Lett. A 240, 185 (1998)

[11] J. H. Shirley, Phys. Rev. 138, B979 (1963)

[12] P. H. Rivera and P. A. Schulz, Proc. $24^{\text {th }}$ Int. Conf. on Phys. of Semicond., Jerusalem, Israel; Ed. David Gershoni, World Scientific, Singapore (1998) 